\title{
On Handovers in Uplink/Downlink Decoupled LTE HetNets
}

\author{
Mukesh Kumar Giluka, M Sibgath Ali Khan, G M Krishna, Touheed Anwar Atif, \\ Vanlin Sathya and Bheemarjuna Reddy Tamma \\ Indian Institute of Technology, Hyderabad, \\ Email: [cs11p1002, ee13p0003, muralikrishnac17, ee13b1036, cs11p1003,tbr]@iith.ac.in
}

\begin{abstract}
Cellular heterogeneous networks (HetNets) are going to be one of the key enablers for 5G. Downlink/Uplink decoupling (DUDe) is a concept in which a mobile device is connected with Macro cell for downlink communication and with small cell for uplink communication in LTE/LTE-A HetNets. It improves uplink data rate, reduces power consumption of devices, balances load between Macro cell and small cells. Due to incorporation of DUDe, a mobile device has to perform separate uplink and downlink handovers unlike traditional handovers in coupled LTE networks. In this paper, we propose various handover schemes for DUDe LTE networks. Apart from this, we have mathematically analysed the received SINR by small cells taken part in decoupling, with respect to a device moving in decoupling regions of these small cells, in multiple cell interference scenario. Simulation results show the signaling impact of DUDe in handovers, increased uplink SINR, decreased power consumption of devices in both single small cell and multiple small cell scenarios.
\end{abstract}

\section{Introduction}

In order to achieve $5 \mathrm{G}$ goals, cellular heterogeneous networks are going to play key role. Presently, cellular network users are not only those which generate mostly downlink (DL) traffic (web browsing, downloading) but also combination of users generating symmetric (both uplink (UL) and downlink) traffic (social networking, gaming) and users generating uplink traffic (IoT devices). These users are not only smartphone, tablet or other mobile computing gadget users but also IoT users which are growing rapidly. In order to reduce the load on Macro cells and to provide better connectivity and high data rate to these users, small cells are being deployed. Presence of such diverse traffic generating users and small cells with different transmit powers and sizes, have turned cellular networks from homogeneous to heterogeneous in nature. In such a heterogeneous environment [?], it is highly possible that a user equipment (UE) or a device will receive signals from different base stations (including Macro cell and small cells) and will have an option to connect with one of them. For a device, a base station which is good in terms of downlink connection, may not be good for uplink connection. This phenomena is termed as UL/DL imbalance. Typically, a better connectivity in uplink/downlink depends on respective received signal strength, interference from other cells, additive white noise, distance of a device from serving base station. In this paper, we have used the terms UE and device interchangeably.
Figure 1 shows that Macro cell is good for UE1 and small cell is good for UE3 for both UL and DL but for UE2, Macro cell is good for DL (due to better downlink signal strength) and small cell is good for UL (lesser path loss in comparison to Macro cell). The concept that a device gets connected to two different cells in downlink and uplink respectively, is called as downlink/uplink decoupling (DUDe). Advantages of decoupling (downlink

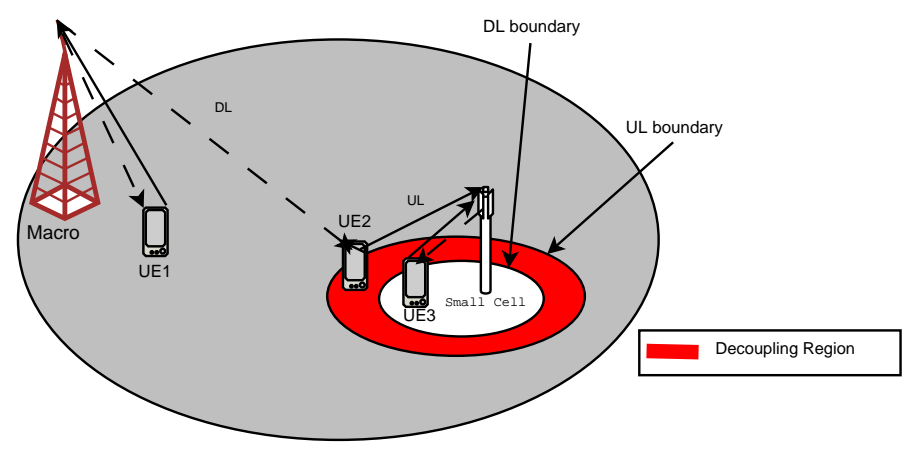

Figure 1. DL/UL Decoupling

with Macro cell and uplink with small cell) are as follows:

- Due to smaller path loss, uplink SNR will increase and transmit power requirement for a device will be lesser for a fixed target SNR.

- Uplink interference condition will be improved due to reduced UL transmit power.

- Increased uplink SNR and decreased uplink interference will result in increased SINR and hence, uplink data rate will be increased.

- UL load on Macro cell can be pushed towards underutilized small cells.

Mobility is a topic in cellular network which always has attracted the attention of researchers because of following issues associated with it: (i) Decrease in throughput of the mobile devices (ii) Excess battery drain (iii) Excess signaling overhead due to positioning signaling and handover signaling. In this paper, we have analysed the effect of mobility on DUDe in both single small cell non-interference scenario and multiple small cells interference scenario.

Organization of the paper is as follows: Section 2 discusses the related work on decoupling. In Section 3, we have proposed various handover schemes in decoupling scenario. Apart from this, we have calculated the gain in SINR due to decoupling. Section 4 presents simulation results and performance analysis of schemes proposed in Section 3. Finally, Section 5 concludes the paper. 


\section{Related Work}

Downlink/Uplink decoupling (DUDe) is a recent proposed advancement in LTE to optimize it for next generation challenges. In [?], authors discussed various advantages of DUDe and shown simulation results to prove their claims. In paper [?], authors compared the performance of DUDe with traditional coupled systems in a static environment with single Macro cell and single small cell. The simulation results are taken in both interference and non-interference environments. Paper [?] analyses the association probability for downlink and uplink in a DUDe system. Authors also studied the decoupled access by increasing the density of small cells with the goal of maximizing the average received power. They proved that the decoupling access will introduce fairness in the uplink throughput. In paper [?], an analytical model is presented for uplink SINR and rate coverage in a K-tier heterogeneous cellular networks with load balancing. Further, the paper defines joint UL/DL rate coverage. Finally, through simulations, it shows that decoupled connection is good for applications which require similar data rate in both UL and DL.

In this paper, we have proposed separate handover schemes for uplink and downlink in the DUDe environment. In the best of our knowledge, this is the first work discussing handovers in UL/DL decoupled LTE HetNets. Apart from this, we have analysed uplink SINR in multiple small cell interference scenario for a mobile device.

\section{Proposed Work}

A mobile UE or device moving from one cell to another performs handover. The decision of handover is taken when downlink SINR received by UE from the serving cell is considerably low in comparison to the target cell. Figure 2 shows a basic traditional handover diagram in which important signaling messages exchange is shown. Here, the UE sends the status (MeasurementReport) of received downlink SINR with respect to both cells, to the serving cell. Based on this measurement report, the serving cell sends handover request (HO Request) to the target cell. After receiving the request, the target cell responses to the serving cell with "HO Response" message. Now, by sending "HO Command" message to the UE, the serving cell instructs it to connect with the target cell and the UE follows the instruction by sending "HO Confirm" message to the target cell.

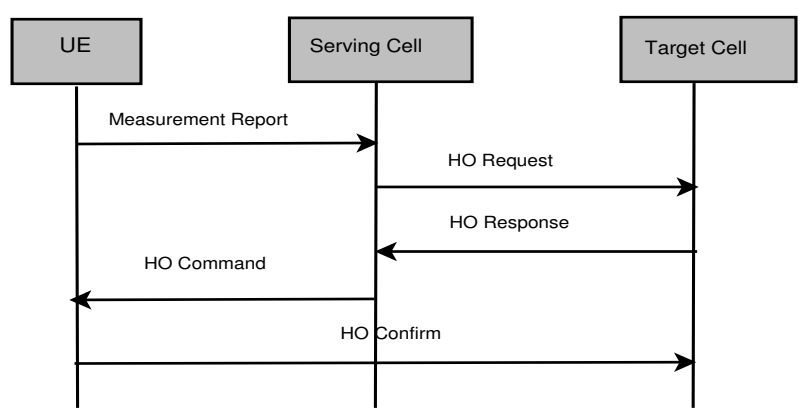

Figure 2. Call Flow Diagram Showing Handovers in Coupled LTE Networks

In this paper, we have considered handover between Macro cell and small cell. As discussed in Section I, in
Figure 1, in the decoupling region (shaded with red color), for a device, Macro cell is good for downlink and small cell is good for uplink communication. Hence, when a device moves from Macro cell region (where both UL and DL connections of the UE are with Macro cell) to small cell region (where both UL and DL connections of the UE are with small cell), it will have to pass through the decoupling region (where UL connection is with small cell and DL connection is with Macro cell). In this case, unlike traditional handover, the device will have to first perform the uplink handover (but not the downlink handover) with small cell as soon as it enters into the decoupling region and then the downlink handover when it enters into small cell region. Similarly, when a device moves from small cell region to Macro cell region, the device will first perform the downlink handover (without the uplink handover) while entering into the decoupling region and then uplink handover. In Figure 3, UE1 is moving from Macro cell region to small cell region following the path $A_{1} B_{1} C_{1} D_{1}$ and UE2 is moving from small cell region to Macro cell region following the path $A_{2} B_{2} C_{2} D_{2} . B_{1}$ and $C_{1}$ are decoupling points (points at which device enters or exits the decoupling region) for UE1 and $B_{2}$ and $C_{2}$ are decoupling points for UE2. $B_{1}$ is also the point where UE1 does uplink handover and $C_{1}$ is the point where it does downlink handover. Similarly, $B_{2}$ is the point where UE2 does downlink handover and $C_{2}$ is the point where it does uplink handover.

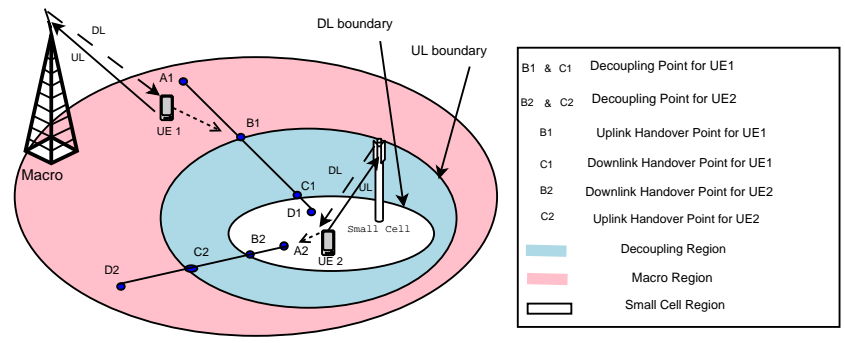

Figure 3. Possible Handover Points in DUDe

The decision for the uplink handover will be taken based on the uplink SINR received by the serving cell and the target cell with respect to the device. Here, the target cell will send its received uplink SINR with respect to the device as "MeasurementReport" to the serving cell. If the received uplink SINR by the target cell is below a threshold then "MeasurementReport" will not be sent by the target cell to the serving cell. After comparing the SINR values of the serving cell and the target cell, the serving cell will initiate handover procedure. For downlink handover, the UE will send the "MeasurementReport" to the serving cell same as the traditional handover scheme. Flow of signaling messages will be different for different cases. There are following four cases possible:

1) Uplink Handover $(U L-H O)$ from Macro cell to small cell

2) Uplink Handover $(U L-H O)$ from small cell to Macro cell

3) Downlink Handover $(D L-H O)$ from small cell to Macro cell

4) Downlink Handover $(\mathrm{DL}-\mathrm{HO})$ from Macro cell to small cell 


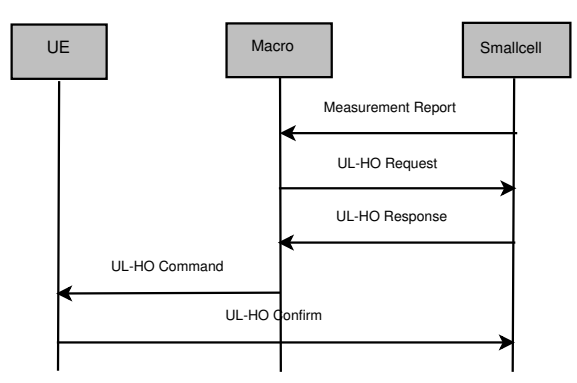

Figure 4. Uplink Handover from Macro cell to Small Cell

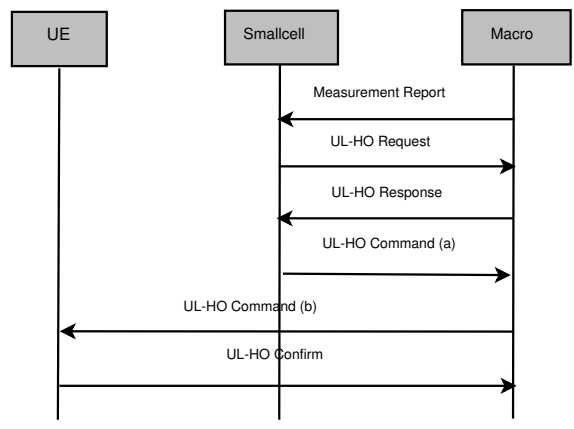

Figure 5. Uplink Handover from Small Cell to Macro cell

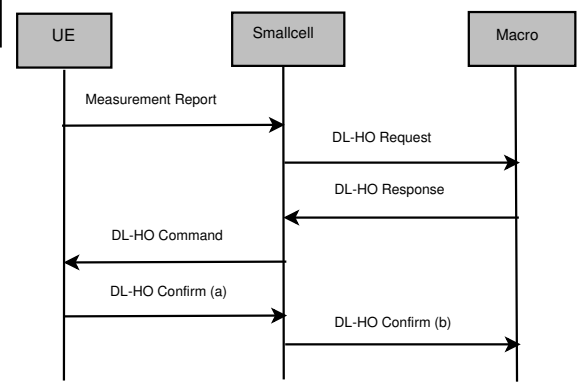

Figure 6. Downlink Handover from Small Cell to Macro cell

\subsection{Uplink Handover from Macro cell to Small Cell}

This situation will occur when a device moves from Macro cell region to small cell region. In the Figure 3, at point $B_{1}$, UE1 will perform uplink handover to small cell whereas downlink will remain connected to Macro cell. In this case, Macro cell will be acting as serving cell and small cell will be acting as target cell. Figure 4 shows the message flow diagram for this case. Small cell (target cell) sends the "MeasurementReport" which contains its SINR value with respect to the device which suppose to perform uplink handover. After receiving this message and comparing with its own received SINR with respect to the device, Macro cell (serving cell) sends " $U L-H O R e q u e s t$ " to small cell as an initiation of the uplink handover process. Now, small cell sends "UL - HOResponse" message to Macro cell as an acknowledgment for being ready for uplink handover. After

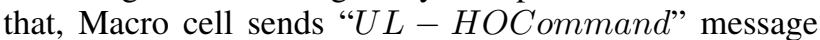
to the device instructing to switch to small cell for uplink connection. By sending " $U L-$ HOConfirm" message to small cell, the device completes the uplink handover process. In order to keep the message flow simple, we have ignored some of the signaling messages.

\subsection{Uplink Handover from Small Cell to Macro cell}

This situation will occur when the device moves from small cell region to Macro cell region. In the Figure 3, at point $C_{2}$, UE2 will perform uplink handover to Macro cell while downlink is already switched to Macro cell. In this case, small cell will be acting as serving cell and The Macro cell will be acting as target cell. Figure 5 shows the message flow diagram for this case. In the figure, " $U L-$ HOCommand" is not directly sent to the device (or UE) but sent via Macro cell because at this point there is no downlink connection exists between small cell and the device. The downlink connection has already been shifted to Macro cell. This is the only difference between Figure 4 and Figure 5.

\subsection{Downlink Handover from Small Cell to Macro cell}

This situation will occur when the device moves from small cell region to Macro cell region. In the Figure 3, at point $B_{2}$, UE2 will perform downlink handover to Macro cell while uplink will remain connected to small cell. In this case, small cell will be acting as serving cell and Macro cell will be acting as target cell. Figure 6 shows the message flow diagram for this case. This message flow is same as the traditional handover mechanism as shown in Figure 2. Here, the term " $H O$ " is replaced with " $D L-$ $H O$ ". In this case, the " $D L-H O C o n$ firm" message is not directly sent to Macro cell but via small cell because at this point, the device has no uplink connection with Macro cell but with small cell.

\subsection{Downlink Handover from Macro cell to Small Cell}

This situation will occur when the device moves from Macro cell region to small cell region. In the Figure 3, at point $C_{1}$, UE1 will perform downlink handover to small cell while uplink is already switched to small cell. In this case, Macro cell will be acting as serving cell and small cell will be acting as target cell. Figure 7 shows the message flow diagram for this case. Here, the "MeasurementReport" is not directly sent to Macro cell but via small cell because at this point, the device has no uplink connection with Macro cell but with small cell.

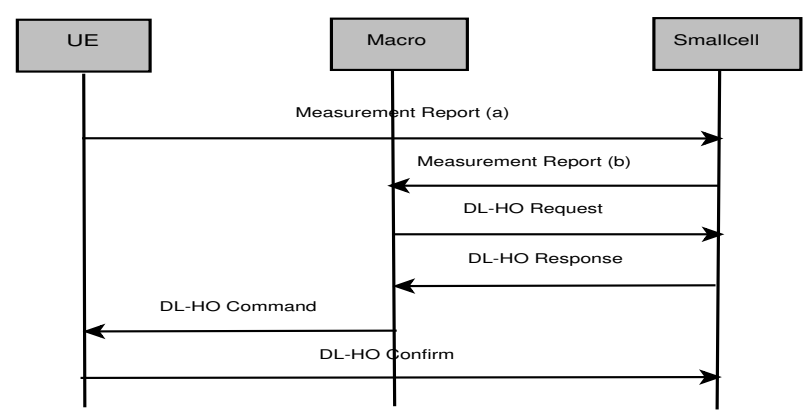

Figure 7. Downlink Handover from Macro cell to Small Cell

Now, let us consider a scenario in which a UE or device does a round trip, starts from the point $A_{1}$ in Macro cell region as shown in Figure 3 and reaches to the same point by following the path $A_{1} B_{1} C_{1} D_{1} D_{1} C_{1} B_{1} A_{1}$. In this journey, in case of coupled connection, the UE will perform two handovers at point $C_{1}$ while in case of decoupled connection, four handovers will be performed (two at point $B_{1}$ and two at point $C_{1}$ ). Therefore, in the latter case, due to two extra handovers, total number of signaling messages exchange in the system will increase. But, after analysing above signaling message flow diagrams of different handovers, it can be concluded that excess signaling consists of mostly uplink signaling message exchange between the UE and small cell which has least impact on system performance because of lesser power consumption by devices (UEs) and lesser signaling overhead on Macro cell.

The concept of DUDe will also be applicable to location management when device is in RRC idle state. In this 
paper, we have looked upon only handover management but not the location management.

\subsection{SINR Analysis in Multiple Small Cell Sce- nario}

In Section I, we discussed basic example of decoupling in a simple cellular heterogeneous environment with one Macro cell and one small cell. In this section, we discuss decoupling in presence of multiple small cells. Figure 8 shows a scenario where one Macro cell and multiple small cells are deployed. The region shaded in red shows the decoupling region with respect to Macro cell and small cell $S_{1}$. Decoupling region with respect to Macro cell and small cell is the area appropriate for DL with Macro cell and UL with small cell. In the figure, section A (coupled connection) shows that UE1 is in decoupling region of Macro cell and small cell $S_{1}$, having both UL and DL connection with Macro cell. Devices UE2 (attached with small cell $S_{2}$ ) and UE3 (attached with small cell $S_{3}$ ) will create interference for Macro cell if they are assigned same resources. Similarly, in Section B (decoupled connection), UE1 is connected to small cell $S_{1}$ in uplink and UE2 and UE3 create interference for $S_{1}$. Now, we will derive equations for received SINR by Macro cell and small cell $\left(S_{1}\right)$ due to UL transmission of the device UE1. Here, interference from devices, located in other small cells, on Macro cell and small cell $S_{1}$ is considered. Here, we have assumed that both Macro

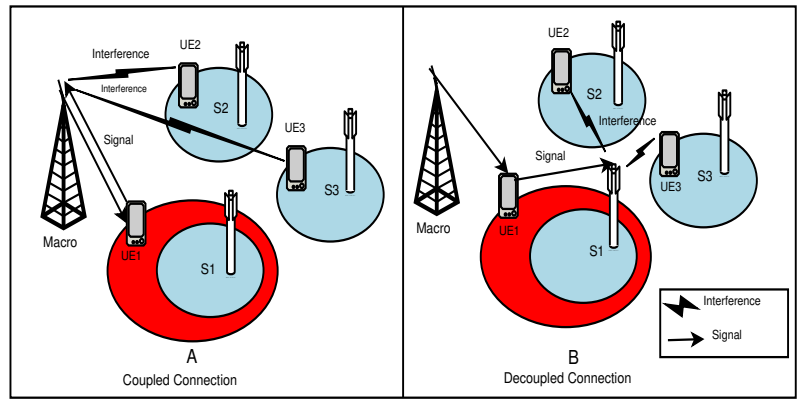

Figure 8. Multiple Small Cells Interference Scenario

cell and small cells have omni-directional antennas and employed open loop fractional path loss power control mechanism. Let, $\alpha$ and $\beta$ are power control factor for Macro cell and small cells respectively. If $P_{T, M}$ is the power with which a device sends its data to Macro cell:

$$
P_{T, M}=\min \left(P_{\max , M}, P_{0, M} \log (N)+P_{0, M}+\alpha P_{L, M}\right)
$$

Where $P_{\max , M}$ is maximum allowed power to transmit, $P_{0, M}$ is the target power which must be received by Macro cell, $N$ is number of resource blocks allocated to the device by Macro cell for UL transmission, $P_{L, M}$ is path loss between the device and Macro cell. For any uplink user in a given cell, $P_{\max }$ is fixed. Hence, we proceed analysis with second term in $P_{T, M}$

$$
P_{T, M}=P_{0, M}+\alpha P_{L, M}
$$

If $P_{R, M}$ is the actual power received by Macro cell then

$$
P_{R, M}=P_{T, M}-P_{L, M}
$$

Hence,

$$
P_{R, M}=P_{0, M}+(\alpha-1) P_{L, M}
$$

Consider $\rho_{G, M}$ as path gain between the device and Macro cell then above equation can be written as

$$
P_{R, M}=\rho_{0, M} \rho_{G, M}^{(1-\alpha)} \text { where, } \rho_{0, M}=10^{\frac{P_{0, M}}{10}}
$$

Received co-channel interference $\left(P_{R, S}\right)$ at Macro cell because of other small cell users allocated the same resource block can be written as following equation. Here, we are assuming that at least one co-channel interference is present in all small cells with respect to device moving from Macro cell to small cell $S_{1}:{ }^{1}$

$$
P_{R, S}=\sum_{i=2}^{K}\left(P_{0, M}+\beta P_{L, S(i)}-P_{L, S(M, i)}\right)
$$

Where, $K$ is the number of small cells, $P_{L, S(i)}$ is the path loss corresponding to a device in $i^{\text {th }}$ small cell and $P_{L, S(M, i)}$ is the path loss corresponding to a device in $i^{\text {th }}$ small cell and Macro cell. In terms of path gain, above equation can be written as following:

$$
P_{R, S}=\sum_{i=2}^{K} \rho_{0, M} \rho_{G, S(i)}^{\beta} \rho_{G, S(M, i)}
$$

Received SINR at Macro cell due to UL transmission of the device UE1:

$$
S I N R_{M}=\frac{\rho_{0, M} \rho_{G, M}^{(1-\alpha)}}{\sum_{i=2}^{K} \rho_{0, M} \rho_{G, S(i)}^{-\beta} \rho_{G, S(M, i)}+N_{0}}
$$

Where $N_{0}$ is the noise factor. Since, the network is densely deployed with small cells:

$$
S I N R_{M}=\frac{\rho_{G, M}^{(1-\alpha)}}{\sum_{i=2}^{K} \rho_{G, S(i)}^{-\beta} \rho_{G, S(M, i)}}
$$

Here, we have ignored short term channel effects and shadowing.

$$
\sum_{i=2}^{K} \rho_{G, S(i)}^{-\beta} \rho_{G, S(M, i)}=L
$$

Therefore,

$$
S I N R_{M}=\frac{\rho_{G, M}^{(1-\alpha)}}{L}
$$

Similarly, SINR received by small cell $S_{1}$ in its decoupling region with Macro cell, from the UL transmission of the device UE1.

$$
S I N R_{S_{1}}=\frac{\rho_{G, S(1)}^{(1-\beta)}}{L}
$$

Consider $\alpha=\beta$, then above two equations can be written in terms of $\alpha$ as follows:

$$
S I N R_{M}=\frac{\rho_{G, M}^{(1-\alpha)}}{L}, \quad S I N R_{S_{1}}=\frac{\rho_{G, S(1)}^{(1-\alpha)}}{L}
$$

1. Here, we have assumed that co-channel interferer is absent between Macro cell and decoupling 
TABLE 1. Simulation Parameters

\begin{tabular}{|l|l|}
\hline \hline Parameters & Values \\
\hline $\begin{array}{l}\text { Macro cell and small cell downlink transmit } \\
\text { power }\end{array}$ & $40,20 \mathrm{dBm}$ \\
\hline Maximum UE uplink transmit power & $23 \mathrm{dBm}$ \\
\hline Number of resource blocks & 10 \\
\hline $\begin{array}{l}\text { Macro cell and small cell power control param- } \\
\text { eter }(\alpha) \text { and }(\beta)\end{array}$ & $0.7,0.7$ \\
\hline Macro cell coverage radius & $1 \mathrm{KM}$ \\
\hline Small cell coverage radius & $0.035 \mathrm{KM}$ \\
\hline Number of Small Cells & 8 \\
\hline Scheduling Algorithm & Round-Robin \\
\hline
\end{tabular}

In terms of distance $d_{M}$ of UE1 from Macro cell and the distance $d_{S_{1}}$ of UE1 from small cell $S_{1}$, above equations can be written as follows:

$$
\begin{aligned}
& \operatorname{SINR}_{M}=\frac{10^{(\alpha-1)\left(35+30 * \log \left(d_{M}\right)\right)}}{L} \\
& S I N R_{S_{1}}=\frac{10^{(\alpha-1)\left(35+30 * \log \left(d_{S_{1}}\right)\right)}}{L}
\end{aligned}
$$

From equations 2 and 3, it can be written that

$$
S I N R_{S_{1}}=\left(\frac{d_{M}}{d_{S_{1}}}\right)^{30(1-\alpha)} * S I N R_{M}
$$

Where $\alpha<1$ and in the decoupling region $d_{M}>d_{S_{1}}$. Above equation shows that in the decoupling region, due to DL/UL decoupling, SINR received by small cell $S_{1}$ will be greater than that of Macro cell by the factor of $\left(\frac{d_{M}}{d_{S_{1}}}\right)^{30(1-\alpha)}$, even after including the interference due to other small cells.

\section{Simulation Results and Analysis}

In this section, we have presented the simulation results to analyse the performance of decoupled connection over coupled connection in the decoupling region for mobility scenario. The section is divided into two subsections, where in the first subsection, results are taken when one Macro cell and single small cell are there. The another subsection discusses the results taken for one Macro cell and multiple small cells. In this scenario, interference due to devices located in different small cells are considered. We have used MATLAB for simulation. Table 4 gives all simulation parameters for both single and multiple small cells scenarios.

\subsection{Single Small Cell Scenario}

4.1.1. Analysis of Impact of Excess Handovers. As discussed in previous section, the considered scenario is to and fro journey of the device following the path $A_{1} B_{1} C_{1} D_{1} D_{1} C_{1} B_{1} A_{1}$ in the Figure 3 . Here, we have assumed that the device always has data to transmit throughout the journey. Here, we are calculating total power consumed by the device in sending data as well as control information including signaling messages involved in handover. Both data and control information will be sent through shared channel. In case of coupled connection, two handovers will be performed by the device. From Figure 2, it can be said that four shared channels will be used by the device to send its signaling messages to perform handover. In case of decoupled connection, in the round trip journey two uplink handovers and two downlink handovers will be performed. From figures 4, 5, 6, 7, it can be said that six shared channels will be used by the device to send its signaling messages to perform handover. We have assumed that number of resource blocks used by a shared channel to send a control information is 50. Power consumed by a device is calculated based on the Equation 1. Figure 12 shows total power consumed per devices in the whole journey. Here, we can see that devices are consuming more power in coupled connection even if they are performing less number of handovers in comparison to decoupling. The reason is that, in coupled connection power consumed by devices to send data is far more because of non-existence of decoupling region.

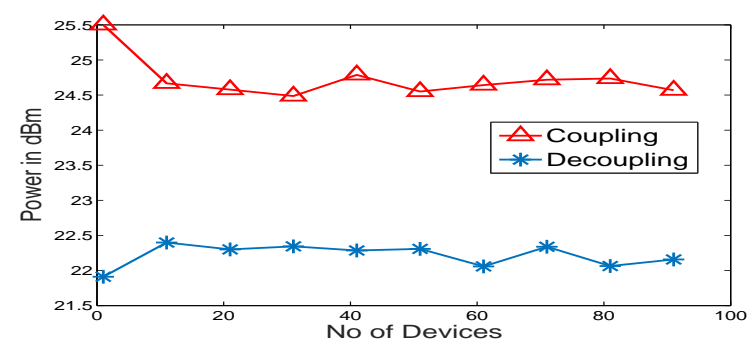

Figure 12. Power Consumption in Round Trip Journey

4.1.2. Improvement in Received SINR and Power Consumption. The considered scenario is shown in Figure 13. The device moves from point ' $A$ ' to point ' $C$ '. At point 'A', the device has coupled connection with Macro cell, i.e., both UL and DL with Macro cell. At point 'B', the decoupling region for the device with respect to small cell starts, i.e., DL with Macro cell and UL with small cell. Point ' $C$ ' is also located in the decoupling region. As per the theoretical basis, if the coupled connection remains uninterrupted after point 'B' then uplink SINR and hence, spectral efficiency will be adversely affected. Figure 9 shows the spectral efficiency curve of the device moving with a particular speed. In the figure, from point 'A' to point 'B', the device is coupled with Macro cell for both UL and DL. The uplink SINR decreases as the device moves away from Macro cell. At point 'B', the decoupling point, the uplink SINR keeps decreasing if coupled connection is maintained but starts increasing if decoupling is done. This happens because in case of decoupling, the device is connected to small cell in uplink which is nearer than Macro cell for the device. Here, in the decoupling region, the device is moving towards small cell, therefore path loss is decreasing which in turn increases the uplink SINR. The speed of the device is taken as $30 \mathrm{KMPH}$.

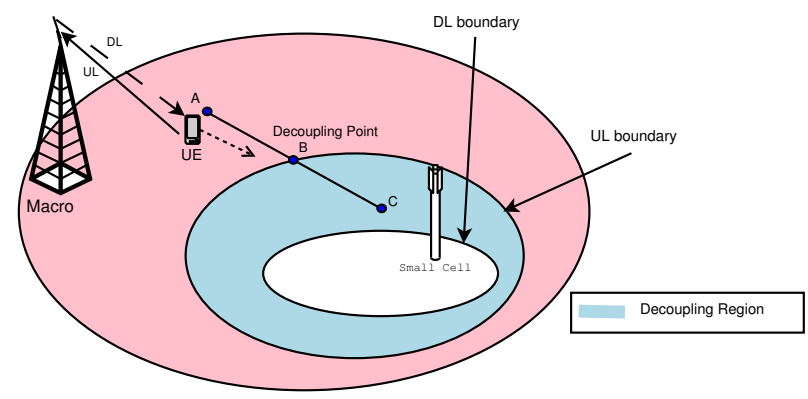

Figure 13. Mobility in Decoupling Region 


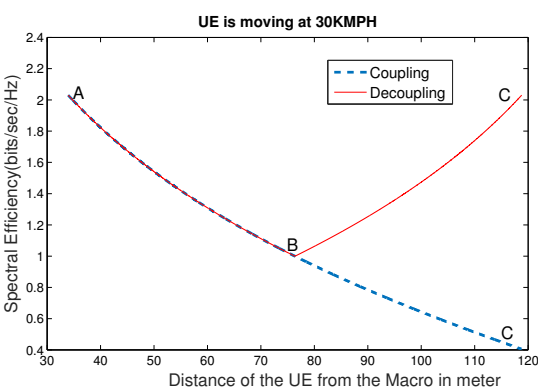

Figure 9. Spectral Efficiency Comparison for Coupling vs Decoupling in Mobility Scenario

Figure 10 shows the power with which the device will have to transmit its signals in order to achieve the uplink SINR of $0 \mathrm{dBm}$. From the starting point 'A' of the movement of the device to the decoupling point ' $\mathrm{B}$ ', the required transmit power increases. After point 'B', it continues to increase for coupled connection upto maximum allowed transmit power of the device which is $23 \mathrm{~dB}$. But, for the decoupled connection, it starts decreasing. In other word, we can say that, in order to achieve same uplink SINR, required transmit power is more in coupled connection in comparison to decoupled connection.

\subsection{Multiple Small Cells Scenario}

Figure 11 shows one Macro cell and multiple small cells scenario. Due to different transmit powers of small cells, decoupling regions will exist in multiple small cell scenario. The device starts its movement from point ' $A$ ' and ends at point 'B'. The red line shows its path from point 'A' to point 'B'. The device always moves in the decoupling region of Macro cell and the respective small cells. Number of small cells are taken as 8 , named as $S_{1}$ to $S_{8}$. Smaller circle with center $S_{1}$ is downlink border and larger circle with center $S_{1}$ is uplink border for small cell $S_{1}$. The area under these two circles is the decoupling region with respect to Macro cell and small cell $S_{1}$. Similarly, decoupling regions of other small cells are drawn. Both, inner and outer circles of a small cell are drawn with same color. Now, we can say that the device will start its journey from point 'A' which is in the decoupling region of Macro cell and small cell $S_{1}$ and will pass through decoupling regions of Macro cell and small cells $S_{2}, S_{3}, S_{4}, S_{5}, S_{6}, S_{7}$ and ends its journey at point 'B' which is in the decoupling region of Macro cell and small cell $S_{8}$. Here, we have kept one device in each small cell to create interference for small cell with which the mobile device is currently attached. These devices are also located in the decoupling region of their respective small cells and represented by blue stars in the figure. So, if the mobile device is currently attached with small cell $S_{1}$, then the remaining static devices (interferer) located in remaining seven small cells will create interference to $S_{1}$ because of which SINR received by $S_{1}$ will be adversely affected.

Figure 14 shows the CDF of uplink SINR received by different small cells. Here, for a particular location of the mobile device, received SINR by all small cells are calculated and the maximum value among all received SINR is termed as uplink SINR of the device at that location. Similarly, SINR at other locations of the mobile device is calculated. In our simulation, SINR is calculated

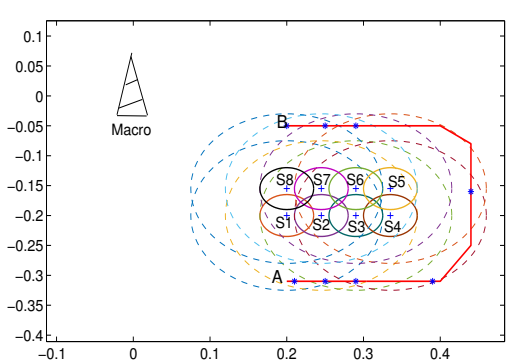

Figure 11. Interference in Multiple Small Cells Scenario

at 100 different locations of the device for both coupled and decoupled connection, throughout its journey from point ' $A$ ' to point ' $B$ '. The figure clearly shows that decoupling always outperforms the coupled connection.

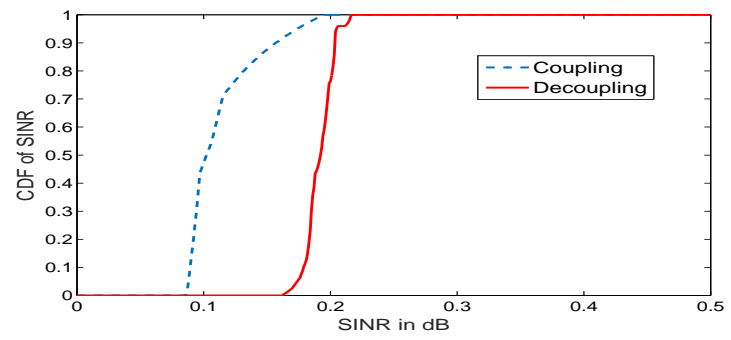

Figure 14. CDF for Uplink SINR

\section{Conclusion}

New handover schemes are proposed and analysed for DUDe scenario which were not existing in coupled scenario. Apart from this, uplink SINR of a device in a multiple cell interference scenario is mathematically analysed. Through simulations, we evaluated the performance of the system in terms of uplink SINR in DUDe environment for both single cell non-interference and multiple cell interference scenario in mobility environment. Results are confirming that DUDe is a better option to increase the efficiency of LTE HetNets.

\section{Acknowlwdgment}

This work was supported by the Deity, Govt of India (Grant No. 13(6)/2010CC\&BT).

\section{References}

